\title{
Oxidative Degradation of Wheat Stalk in Sodium Hypochlorite Solution
}

\author{
Nannan Xia, Guizhen Gong
}

(School of Chemical Engineering, Xuzhou University of technology, Xuzhou 221018, Jiangsu

Province )

\begin{abstract}
The oxidative degradation of the wheat straw was carried out with $\mathrm{NaOCl}$ aqueous solution. The resulting oxidative degradation solution was fractionated and the extract was analyzed by FTIR. The results show that a large amount of oxygen - containing degradation products can be extracted by ether and ethyl acetate, and some products containing $\mathrm{C}-\mathrm{H}$ and $\mathrm{C}=\mathrm{C}$ can be effectively enriched by petroleum ether and $\mathrm{CS}_{2}$. It also shows that the main components of wheat straw, hemicellulose and lignin in $\mathrm{NaOCl}$ aqueous solution can be effectively degraded into small molecule compounds. This study provides theoretical basis and technical support for obtaining high value-added chemicals from wheat straw.
\end{abstract}

Keywords: Wheat Straw; Sodium Hypochlorite; Oxidation; FTIR Analysis

\section{麦秆在次氯酸钠水溶液中的氧化降解}

\author{
夏楠楠，宫贵贞*
}

摘要: 用 $\mathrm{NaOCl}$ 水溶液将麦秆进行氧化降解, 所得的氧化降解溶液进行分级萃取, 萃取物用 FTIR 进行分析。结果表 明：大量含氧的降解产物可被乙醚和乙酸乙酯萃取，而一些含 $\mathrm{C}-\mathrm{H}, \mathrm{C}=\mathrm{C}$ 的产物可被石油醚和 $\mathrm{CS}_{2}$ 有效富集。也说明麦秆 中的主要成分纤维素、半纤维素和木质素在 $\mathrm{NaOCl}$ 水溶液中能有效降解为小分子化合物。该研究为从麦秆中获得高附加值 的化学品提供理论依据和技术支撑。

关键词：麦秆；次氯酸钠；氧化；FTIR 分析

中图分类号：TQ353.9 文献标志码：A

\section{引言}

目前为止人类获取能源的主要方式依然是化石燃料, 但是随着世界工业革命的发生以及世界经济的快 速增长, 对能源的利用和依赖的要求越来越高, 从而使得现在化石能源的价格一直在变得越来越高, 同时 因为能源有限, 地球上的可利用化石能源变得越来越少, 并且化石能源是不可再生的资源。化石能源在开 采、利用过程中导致释放越来越多的二氧化碳气体到地球的大气层中, 引发越来越严重的温室效应, 是地 球面临愈发严峻的生态危机。因此, 寻求可再生的环保的新能源是解决能源危机的关键所在。

生物质是一切可以直接利用或者间接利用绿色植物的光合作用来形成的可以再生或可以循环的有机 质, 主要来源于动植物, 微生物以及它们产生的代谢物 [1]。生物质能源是一种能够储存在生物质体内的 由太阳能转化而来的能量 [2]。因为生物质不会增加大气中的二氧化碳, 其含有较少的硫和氮 [3], 可看作 清洁的能源。所以生物质能源具有非常广阔的发展前景。然而据研究表明, 现在地球上能够作为能源来利 用的生物质能源仅仅占到生物质能源总量的 3\%[4]。农作物秸秆产量巨大，但目前国内外对其利用技术还 处在初级阶段, 总的来说利用技术还不够成熟, 大概可以归纳为 4 种利用技术: 秸秆还田技术, 能源化技 术，饲料化技术和工业化利用技术 $[5-8]$ 。 
次氯酸钠便宜易得常用于天然物质的氧化降解 $[9,10]$, 因此，本文考察了次氯酸钠对麦秆的氧化降 解，并用四种有机溶剂进行萃取，以其为麦秆的高附加值利用提供理论依据和技术支撑。

\section{1 实验部分}

\section{1 原料、试剂和仪器}

实验选取江苏省徐州市农作物成熟后所收的麦秆作为原料样品。将麦秆用去离子水洗涤干净，再在阳 光下面晒干。使用粉碎机把麦秆样品进行粉碎, 然后使用 80 目篮进行篎选, 放在 $70^{\circ} \mathrm{C}$ 的真空干燥箱中 干燥 $24 \mathrm{~h}$, 最后把麦秆样品放在干燥器中备用。所用试剂石油醚、乙醚、二硫化碳及乙酸乙酯均为分析纯 试剂, 所有有机溶剂均经旋转蒸发仪蒸馏后使用。

上海亚荣生化仪器厂生产的 RE52CS-1 型旋转蒸发仪; 巩义市予华仪器有限责任公司 DF-101S 集热式 恒温加热磁力摚拌器; 上海贺德实验设备有限公司生产的 DZF6020 型真空干燥箱; ALPHA 型傅立叶转换红 外光谱仪（FTIR，德国 Bruker 公司）。

\section{2 实验方法}

称取干燥的麦秆样品 $5.0 \mathrm{~g}$, 将样品放入到 $250 \mathrm{~m} 1$ 的烧瓶中, 再加入 $\mathrm{NaOCl}$ 水溶液 $50 \mathrm{ml}$, 水浴加 热到 $40{ }^{\circ} \mathrm{C}$, 恒温连续磁力摚拌反应 24 小时。反应毕, 冷至室温, 将反应所得产物进行抽滤, 得滤液 1 及 残渣。过滤所得滤渣放到真空干燥箱中于 $70{ }^{\circ} \mathrm{C}$ 燥至恒重, 称重; 所得的滤液依次用石油醚, $\mathrm{CS}_{2}$, 乙醚, 乙酸乙酯四种有机溶剂进行分级萃取。所得萃取液用旋转蒸发仪蒸馏, 得萃取液。最后一级的萃余液使用 盐酸酸化, 调节 PH 至 2-3。经盐酸酸化过的产物再次过滤, 得到滤液 2 和滤渣。滤液进一步用石油醚, $\mathrm{CS}_{2}$, 乙醚, 乙酸乙酯四种有机溶剂进行分级萃取。所得萃取液用 KBr 压片进行 FTIR 分析, 在 $400^{\sim} 4000 \mathrm{~cm}^{-1}$ 波 数范围内扫描。

\section{2 结果与讨论}

如图 1 所示为滤液 1 各级萃取液的 FTIR。在 $3412 \mathrm{~cm}^{-1}$ 特征吸收谱带附近有较强的 0-H 键特征吸收。 四种萃取物中乙醚和乙酸乙酯萃取物表现出了明显的 $0-\mathrm{H}$ 键特征吸收谱, $\mathrm{CS}_{2}$ 萃取物有一尖的吸收, 而石油 醚则没有明显的吸收峰。 $2929 \mathrm{~cm}^{-1}, 2850 \mathrm{~cm}^{-1}$ 是 $\mathrm{CH}_{3}$-和 $-\mathrm{CH}_{2}$-的伸缩振动峰, 乙酸乙酯此处吸收峰最明显, 石油醚和 $\mathrm{CS}_{2}$ 次之, 乙醚中该吸收较弱。1736 $\mathrm{cm}^{-1}$ 特征吸收谱带为羰基的吸收峰, 图中四萃取物中唯一有 明显特征频率的是乙酸乙酯萃取物, 另外三种则没有相应的特征吸收峰。 $\mathrm{CS}_{2}$ 萃取物在 $1627 \mathrm{~cm}^{-1}$ 附近有强 烈的吸收, 此处是 $\mathrm{C}=\mathrm{C}$ 不饱和键特征吸收谱带, 乙醚次之, 乙酸乙酯又弱于乙醚, 石油醚中不明显。1217 $\mathrm{cm}^{-1}$ 和 $1090 \mathrm{~cm}^{-1}$ 两处为 $\mathrm{C}-0$ 的吸收, 乙酸乙酯中吸收最强。 $838 \mathrm{~cm}^{-1}$ 附近的吸收为取代苯环的特征峰, 表明木 质素发生了降解生成小分子物质。由以上分析可知, 乙醚和乙酸乙酯能从秸秆的氧化降解液中萃取出一些 含氧的极性物质，而一些含 $\mathrm{C}-\mathrm{H}$ 的产物可被石油醚和 $\mathrm{CS}_{2}$ 富集。 


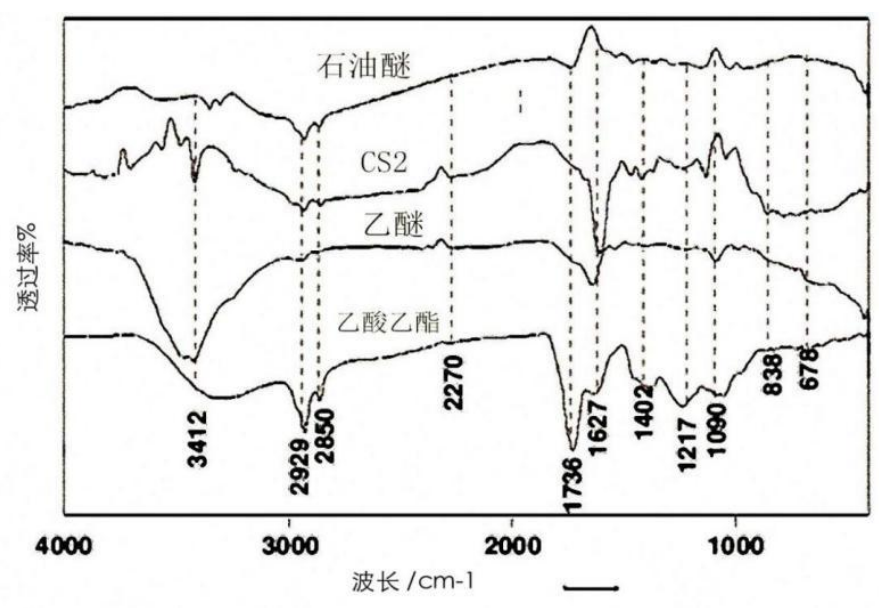

图 1 滤液 1 中各萃取物的 FTIR 分析

滤液 1 经四种有机溶剂萃取后用盐酸酸化, 滤液里以钠盐形式存在的物质变为酸的形式。将滤液 2 进 一步分级萃取, 如图 2 所示为各级萃取液的 FTIR。由图可知, 乙酸乙酯萃取物在 $3412 \mathrm{~cm}^{-1}$ 有一宽的强吸 收, 表明有大量的缔合羟基存在, 石油醚中此处峰行尖锐, 说明石油醚也萃取出一些含羟基物质, 以游离 状态存在。在 $1736 \mathrm{~cm}^{-1}$ 附近显示的 $\mathrm{C}-0$ 吸收谱带, 强度明显强于未酸化前的萃取物, 说明未酸化前, 以 钠盐形式存在, 大多物质难以被萃取出, 酸化后萃取率大大提高。同时在 $838 \mathrm{~cm}^{-1}$ 附近的取代苯吸收峰, 在乙醚中也明显强于未酸化前。

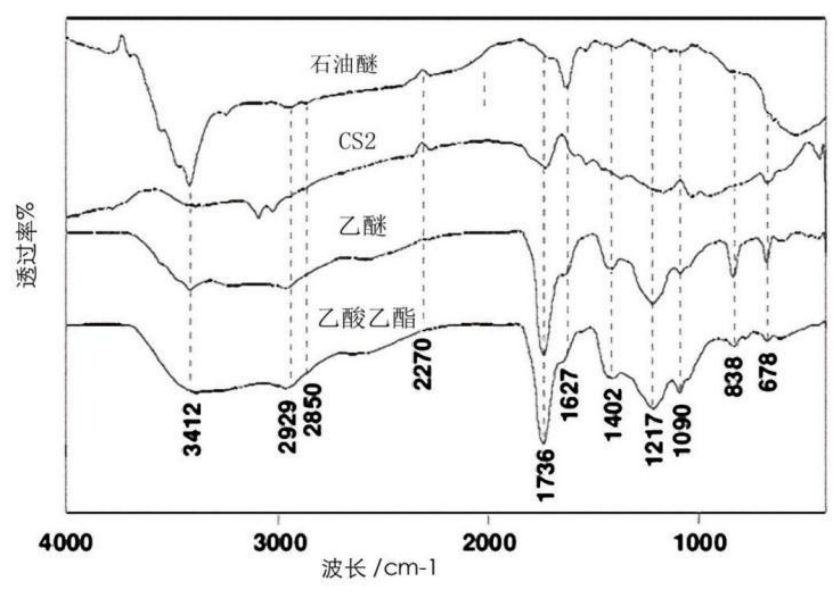

图 2 滤液 2 中各萃取物的 FTIR 分析

\section{3 结论}

本研究考察了麦秆在 $\mathrm{NaOCl}$ 水溶液中的氧化降解, 对氧化降解所得的产物进行分级萃取, 并用 FTIR 进行分析。根据图谱分析可知, 麦秆中的主要成分纤维素、半纤维素和木质素在 NaOC1 水溶液中能有效降 解。大量含氧的降解产物可被乙醚和乙酸乙酯萃取, 而一些含 $\mathrm{C}-\mathrm{H}, \mathrm{C}=\mathrm{C}$ 的产物可被石油醚和 $\mathrm{CS}_{2}$ 有效富集。

\section{4 致谢}

感谢中国建筑材料联合会（2014-M3-4），徐州情报所课题（XKQ016）的资助！

\section{Acknowledgement}

This work was supported by the China Building Material Federation (2014-M3-4) and Xuzhou Information Institute (XKQ016). 


\section{参考文献:}

[1] 王庆, 王英勇, 郭向云. 生物形态的高性能材料 [J]. 化学进展, 2007, 19 (7-8): 1217-1222.

[2] 王小孟, 谭江林, 陈金珠. 我国生物质能源开发利用的现状 [J]. 江西林业科技, 2006, (5): 45-57.

[3] 赖艳华, 吕明新, 马春元, 等. 秥秆类生物质热解特性及其动力学研究 [J]. 太阳能学报, 2002, 23 (2): 203-206.

[4] 朱锡锋, 朱建萍. 生物质热解液化技术经济分析 [J]. 新能源及工艺, 2006 (6): 32-34.

[5] 匡廷云, 白克智, 卢从明, 等. 生物质能源技术前瞻 [J]. 太阳能, 2004, (4): 7-9.

[6] 陈羲, 韩志群, 孔繁华, 等. 生物质能源的开发与利用 [J]. 化学进展, 2007, 19 (7-8): 1091-1097.

[7] 马隆龙, 吴创之, 孙立. 生物质气化技术及其应用 [M]. 北京: 化学工业出版社, 2003.

[8] 何方，王华. 生物质液化制取液体燃料和化学品 [J]. 能源工程, 1999, (5): 14-17.

[9] MAYO F R. Application of sodium hypochlotite oxidations to the structure of coal [J]. Fuel, 1975, 54 (4): 273-275.

[10] KANAZAWA H, ONAMI T. Mechanism of the degradation of organge $\mathrm{G}$ by sodium hypochlorite [J]. Color Technol., 2001, 117: 323-327.

\section{References:}

[1] Wang Qing, Wang Yingyong, Guo Xiangyun. High-Performance Materials of Biological Form [J]. Chemical Progress, 2007, 19 (7-8): 1217-1222.

[2] Wang Xiaomeng, Tan Jianglin, Chen Jinzhu. Status Quo of Exploitation and Utilization of Biomass Energy in China [J]. Journal of Jiangxi Forestry Science and Technology, 2006, (5): 45-57.

[3] REN Yanhua, LU Mingxin, MA Chunyuan, et al. Pyrolysis Characteristics and Kinetics of Straw Biomass [J]. Journal of Solar Energy, 2002, 23(2): 203-206.

[4] ZHU Xi - feng, ZHU Jian - ping.Economic Analysis of Biomass Pyrolysis Liquefaction Technology [J]. New Energy and Technology, 2006 (6): 32-34.

[5] Kuang Tingyun, Bai Kezhi, Lu Congming, et al. Prospects for Biomass Energy Technology [J]. Solar energy, 2004, (4): 7-9.

[6] Chen Xi, Han Zhiqun, Kong Fanhua, et al. Development and Utilization of Biomass Energy [J]. Chemical Progress, 2007, 19 (7-8): 1091-1097.

[7] Ma Longlong, Wu Chongzhi, Sun Li. Biomass Gasification Technology and its Application [M]. Beijing: Chemical Industry Press, 2003.

[8] He Fang, Wang Hua. Biomass Liquefaction for Liquid Fuels and Chemicals [J]. Energy Engineering, 1999, (5): 14-17.

[9] MAYO F R. Application of sodium hypochlotite oxidations to the structure of coal [J]. Fuel, 1975, 54 (4): 273-275.

[10] KANAZAWA H, ONAMI T. Mechanism of the degradation of organge $\mathrm{G}$ by sodium hypochlorite [J]. Color Technol., 2001, 117: 323-327. 